\title{
Neue Aufgaben des Destinationsmanagements: Zwischen Tourismus und Lebensqualität
}

\author{
Christl Wittmann ${ }^{1,2} \mathbb{D} \cdot$ Lena Helleisz ${ }^{3}$ \\ Eingegangen: 30. September 2021 / Überarbeitet: 27. Januar 2022 / Angenommen: 28. Januar 2022 / Online publiziert: 7. März 2022 \\ () Der/die Autor(en) 2022
}

\section{Zusammenfassung}

Dieser Artikel soll die Notwendigkeit einer neuen Sichtweise in der Tourismusentwicklung darlegen: Die Planung sollte sich weg vom aktuell oft vorhandenen rein wirtschaftlichen Wachstumsgedanken entwickeln, hin zu einem integrativen Planungsansatz, der auch die Bedürfnisse der vor Ort wohnenden Bevölkerung miteinbezieht. Durch eine Annäherung an den Begriff des „Lebensraummanagements“ wird in diesem Beitrag aufgezeigt, welche Aspekte und Aufgaben Destinationsmanagementorganisationen (DMO) dabei berücksichtigen sollten und wie eine praktische Implementierung aussehen kann. Am Beispiel des im Süden Hessens liegenden Main-Kinzig-Kreises (MKK) wird im Anschluss gezeigt, welche Faktoren einen Landkreis oder eine DMO zu diesem Schritt veranlassen. Der MKK hat erste Schritte in Richtung eines Perspektivwechsels getan und die lokale DMO auch mit Aspekten des Standortmarketings betraut. An diesem Beispiel wird verdeutlicht, welche Synergien bei der Umsetzung genutzt und welche Vorteile aus dieser Herangehensweise für die Region resultieren können. Besonders im Hinblick auf die aktuelle Nachhaltigkeitsdebatte im Tourismus ist der Wandel als Chance zu sehen.

Schlüsselwörter Tourismusentwicklung $\cdot$ Lebensraum $\cdot$ Integrierte Planung $\cdot$ Spessart $\cdot$ DMO

\begin{abstract}
This article shows the necessity for a new perspective in tourism development: Planning should develop away from the (often predominant) purely economic growth and towards a more integrated planning approach that also considers the needs of the local inhabitants. By becoming closer to the term "living environment management", it is shown in this article which aspects and tasks destination management organizations (DMOs) should take into consideration and what practical implementation can look like. The example of Main-Kinzig-Kreis (MKK), located in southern Hesse in Germany, shows which factors can lead an administrative district or DMO to this step. MKK took the first steps in the direction of a change of perspective and entrusted the local DMO with aspects of local marketing. This example shows which synergies can be used during implementation and which benefits for the region can result from this approach. Especially with regard to the current discussion of sustainability in tourism this change can be seen as an opportunity.
\end{abstract}

Keywords Tourism development $\cdot$ Living environment $\cdot$ Integrated planning $\cdot$ Spessart $\cdot$ DMO

$\triangle$ Christl Wittmann

christl.wittmann@hs-heilbronn.de

Lena Helleisz

lena.helleisz@kohl-partner.eu

Hochschule Heilbronn, Heilbronn, Deutschland

2 Spessart Tourismus und Marketing $\mathrm{GmbH}$, Holzgasse 1, 63571 Gelnhausen, Deutschland

3 Kohl \& Partner Stuttgart, Auf der Höhe 42, 73529 Schwäbisch Gmünd, Deutschland

\section{Einleitung}

Aktuelle Debatten über Overtourism, Tragfähigkeit und Tourismusakzeptanz lassen erahnen, dass ein Umbruch im Destinationsmanagement bevorsteht: Weg vom bisher häufig vorherrschenden Wachstumsfokus, hin zu einer integrierten Tourismusplanung und -entwicklung, welche die Belange und die Lebensqualität der lokalen Gesellschaft miteinbezieht (Uğur 2017; Milano et al. 2019). Mit diesem Blick würde dem Faktor Rechnung getragen, dass sich 
Bewohner*innen und Gäste in einer Destination einen geografischen Raum teilen, wodurch eine Balance zwischen den Bedürfnissen der verschiedenen Anspruchsgruppen notwendig ist (Uğur 2017). Die Tendenz neuer Strategien geht also hin zu einem ganzheitlichen Blick, der nicht nur berücksichtigt, was für den Gast gut ist, sondern die Frage nach dem Mehrwert für die gesamte Region stellt. Die Basis für diesen Perspektivwechsel bildet dabei das Bewusstsein, dass die Destination und die Reiseerfahrung nicht nur von natürlichen Gegebenheiten und der (touristischen) Infrastruktur abhängen, sondern vor allem auch von den Bewohner*innen und den Gast-Gastgebende-Begegnungen, denn ,ist der Einheimische nicht mehr gewillt seinen persönlichen Lebensraum mit Fremden $\mathrm{zu}$ teilen, kann das touristische Angebot nicht glaubwürdig gestaltet werden“ (Hölzl 2019, S. 253). Diese Aussage zeigt die Notwendigkeit, Gedanken zum „Lebensraum“ in die Tourismusentwicklung einfließen zu lassen.

\section{Annäherung an „Lebensraum" und "Lebensqualität"}

Eine allgemeingültige Abgrenzung des Begriffs „Lebensraum“ existiert bisher nicht. Sowohl die Definition des Duden (2021) als ,Raum, Umkreis, in dem sich jemand oder eine Gemeinschaft (frei) bewegen und entfalten kann“ wie auch die Formulierung ,Tourismus für lebenswerte Regionen“ des Kompetenzzentrum Tourismus des Bundes (2019) rücken jedoch das Thema „Lebensqualität“ in den Fokus. Auch dieser Begriff wird bisher nicht über allgemeingültige Faktoren definiert, da diese gemeinhin als subjekti-

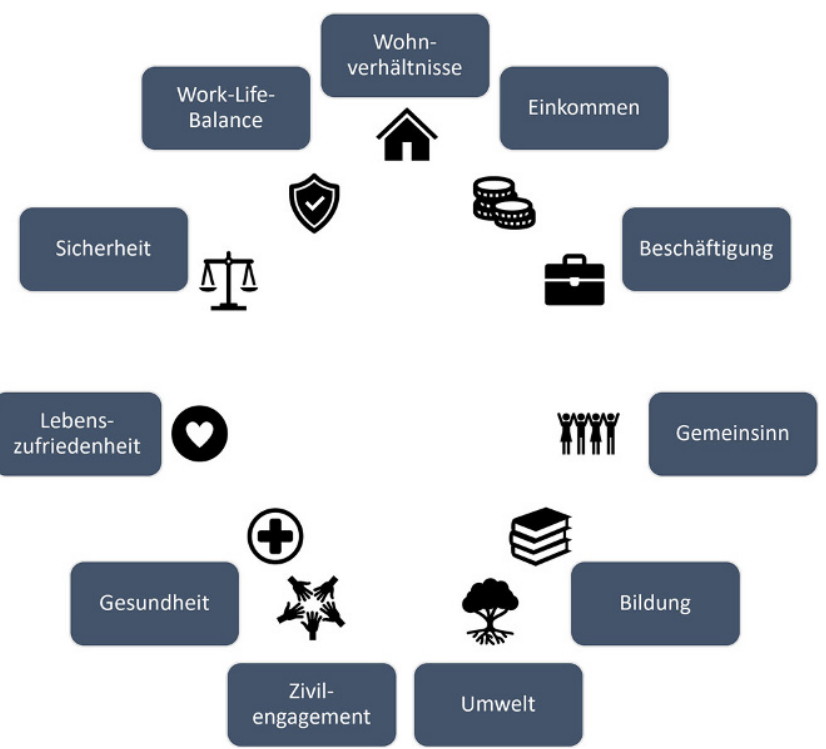

Abb. 1 Faktoren der Lebensqualität, eigene Darstellung basierend auf OECD (o.J.) ve Wahrnehmung eines Individuums gilt, welche stark von der jeweiligen Lebenssituation sowie dem eigenen Umfeld abhängig ist (World Health Organization 1997). Ein exemplarisches Indikatorenset aus der „Better Life Index“Erhebung der Organisation für wirtschaftliche Zusammenarbeit und Entwicklung (OECD) (OECD o.J.) ist in Abb. 1 dargestellt.

Für den Tourismus kann somit als Annäherung definiert werden, dass das Lebensraummanagement Aspekte beinhaltet, welche auf die Lebensqualität der Bewohner*innen in deren geographischen Aufenthaltsraum einzahlt. Bisherige Lebensraumkonzepte - z.B. aus Norderney (Kohl und Partner 2020a) und Bad Hindelang (Kohl und Partner 2020b) - zeigen bereits, dass folgenden Handlungsfeldern besondere Relevanz zukommt:

- Verkehr und Mobilität

- Gemeinschaft und Identität

- Besucherlenkung

- Regionale Wertschöpfung und Wirtschaft

- Employer Branding

- Angebote und Infrastruktur für Einheimische

- Naturschutz und ökologische Nachhaltigkeit

- Begegnungsqualität

- Kommunikation nach innen

- Wohnen

Klar ist, dass die Priorisierungen der einzelnen Handlungsfelder nicht in jeder Destination gleich sind, sondern stark von den lokalen Gegebenheiten und dem Entwicklungsstand des Tourismus abhängen.

\section{Die Rolle der Destinationsmanagementorga- nisation (DMO)}

Das Lebensraummanagement ist dabei keine zwingende Rolle der DMO, vor allem, wenn bereits eine Organisation besteht, welche mit der Regionalentwicklung betraut ist - doch dies ist in vielen Regionen bisher nicht der Fall. Die DMO als interdisziplinär vernetzte Organisation ist für diese Aufgaben prädestiniert, da sie sich bereits um viele der obigen Handlungsfelder kümmert, wenn auch meist mit dem Fokus auf Gästebedürfnisse. Somit liegt es nahe, diese bestehenden Netzwerke zu nutzen und auszubauen und die bisherige Sichtweise um den Blickwinkel der Einheimischen zu erweitern. Durch die Bündelung dieser Aufgaben in einer Organisation kann außerdem eine gute Abstimmung der Entwicklungen mit Blick auf die notwendige Balance zwischen Einheimischen und Gästen geschaffen werden.

Deutlich wird bei dem Blick auf die Breite an Aufgaben jedoch, dass eine DMO diese Aufgaben weder mit ihren Ressourcen noch mit ihrem bestehenden Know-how stem- 
men kann. Vielmehr sollte sich die DMO als „Netzwerk der Netzwerke“ und somit als Initiatorin dieser regionalen Entwicklungen sehen, die stark mit lokalen und regionalen Partner*innen kooperiert (Kohl und Partner 2016). Wünschenswert wäre hier die Schaffung agiler und interdisziplinärer Strukturen, die durch die DMO gesteuert und koordiniert werden, jedoch von verschiedenen Partner*innen in der Region unterstützt werden.

\section{Vorteile für Regionen}

Von der Bündelung und gezielten Bearbeitung der genannten Handlungsfelder kann die gesamte Region profitieren, da ihre Attraktivität steigt. Damit können auch positive Effekte für den Tourismus geschaffen werden: Attraktive Lebensräume sind häufig auch attraktive Urlaubsräume. So ist beispielsweise die Förderung einer positiven regionalen Identität für den Tourismus von Interesse, da durch das hierdurch generierte positive Bild der eigenen Region (Pfeffer-Hoffmann 2011) auch die Gast-Gastgebende-Beziehung profitieren kann. Durch eine positive Kommunikation der regionalen Besonderheiten oder die Einbindung regionaler Kulturangebote trägt der Tourismus bereits zu einer regionalen Identität bei. Noch gezielter kann dies z.B. durch Angebote, die konkret an die Einwohner*innen adressiert sind und deren Kultur und Eigenheiten in den Fokus stellen, gefördert werden. Ein weiteres, häufig genanntes Thema ist die regionale Wertschöpfung, die oft als positiver Effekt touristischer Entwicklung wahrgenommen wird (Seeler et al. 2021). Die hierdurch generierten positiven Effekte auf die gesamte Region und auf individuelle Lebensbereiche können dabei zur Tourismusakzeptanz beitragen. Ein verbreiteter Ansatz ist der Aufbau von Regionalmarken zur Stärkung regionaler Produkte und Angebote, wodurch auch gegenüber dem Gast ein Qualitätsversprechen abgegeben wird. Eine thematische Erweiterung dieser Marken ist die Etablierung einer gemeinsamen Arbeitgebermarke im Sinne einer „Employer Destination“, um auch hier Standards zu setzen und die Reichweite zu erhöhen, wie es z.B. in der Eifel umgesetzt wird (Regionalmarke EIFEL GmbH o.J.).

Diese exemplarisch genannten Handlungsfelder bieten eine Fülle an Aufgaben und Möglichkeiten, um die Themen des Lebensraummanagements in die Arbeit der DMO $\mathrm{zu}$ integrieren und dort Synergien zu nutzen, wobei häufig positive Effekte für Tourismus und Gäste geschaffen werden können. Die Basis für ein solches Vorhaben sollte dabei immer die breite und offene Beteiligung sein, um dringende Themen zu identifizieren und Lösungen zu schaffen, die von den Bewohner*innen und Akteur*innen der Destination angenommen und unterstützt werden - ganz im Sinne des Ansatzes der Destination Governance (Steinecke und Herntrei 2017; Fürst 2013).

\section{Ganzheitlicheres Marketing der Region im Main-Kinzig-Kreis}

Das beschriebene Umdenken hin zu einem umfassenderen, integrativen Planungsansatz äußert sich z. B. in verstärktem Stadt- oder Standortmarketing. Im Folgenden soll nun ein solcher Ansatz vorgestellt werden: Durch die in den letzten Jahren positiven Entwicklungen bei der Bevölkerung und auf dem Arbeitsmarkt (Main-Kinzig-Kreis 2020) lässt sich schließen, dass sich der südhessische Main-Kinzig-Kreis (MKK) gut als Lebens- und Arbeitsort eignet. Deshalb hat er die lokale DMO beauftragt, die Region als Wirtschafts-, Arbeits- und Lebensstandort zu vermarkten und gezielt um Fachkräfte und neue Bewohner*innen für die Region zu werben. Somit stellt er ein gutes Fallbeispiel für diesen Artikel dar.

Als attraktiver Lebensraum mit ausgebauter Freizeitinfrastruktur, guter Anbindung und ansprechenden Angeboten kann die Region auch als Arbeitsraum punkten, da durch die steigende Bedeutung der Work-Life-Balance diesen weichen Standortfaktoren ein wachsender Stellenwert zukommt. Die hohe Lebensqualität und andere weiche Standortfaktoren sollen verstärkt im Marketing genutzt werden, um weitere Fachkräfte und Einwohner*innen in die Region zu holen, wodurch der Landkreis wettbewerbsfähig und lebenswert bleibt. Diese Aufgabe wurde der lokalen DMO, der Spessart Tourismus und Marketing GmbH (STM), übertragen.

\section{Die Spessart Tourismus und Marketing GmbH}

Die Spessart Tourismus und Marketing GmbH ist eine 2016 gegründete DMO mit der Aufgabe, den hessischen Spessart und den MKK zu vermarkten (STM 2021). Die Grundzüge des Tourismusmarketings wurden im Zuge der Gründung erarbeitet und in einem Strategischen Entwicklungsplan (STM 2017) festgehalten, der als Orientierungshilfe für die Tourismusentwicklung dient. Das Tourismusmarketing bei der STM umfasst vor allem Marketing-, Koordinations- und Netzwerkaufgaben.

Obwohl bei DMOs das Marketing auf potenzielle Besucher*innen ausgerichtet ist, galt von Beginn der Grundsatz, dass was für Gäste von Interesse ist, auch für Bewohner*innen von Interesse ist. Dementsprechend ist das Marketing der STM in gleichem Maße an die Bewohner*innen gerichtet, um auf die Angebote am eigenen Wohnort (z.B. die Landschaft oder die Wasserbüffel bei Erlensee; siehe Abb. 2 und 3) und damit die Lebensqualität aufmerksam zu machen.

In den ersten Jahren nach der Gründung lag der Fokus auf Tourismusmarketing, dann wurden Teilaspekte des Standortmarketings als Aufgabe übernommen (STM 2021). Die Arbeit der STM ist hier vor allem inspirativer Natur: 


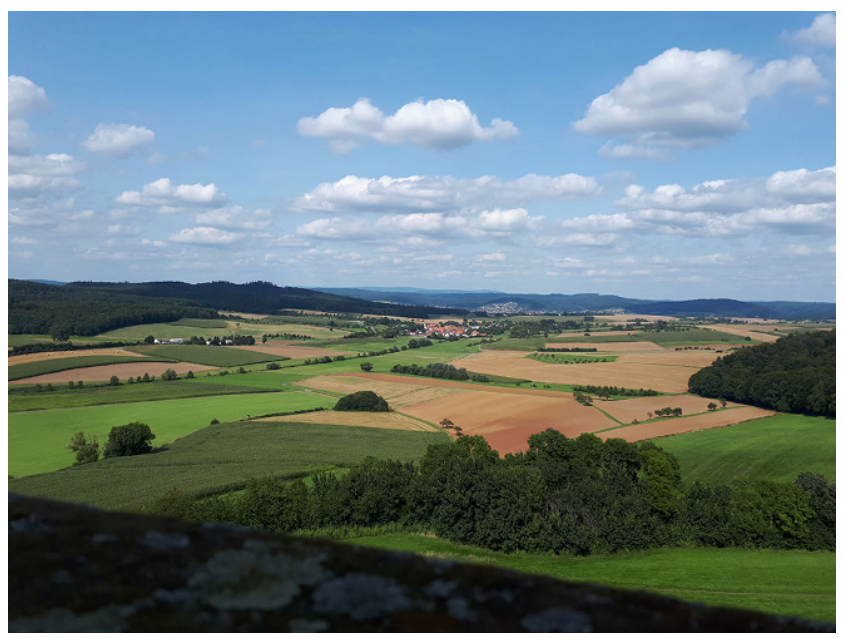

Abb. 2 Ausblick in die Landschaft um die Gemeinde Ronneburg (C) Christl Wittmann)

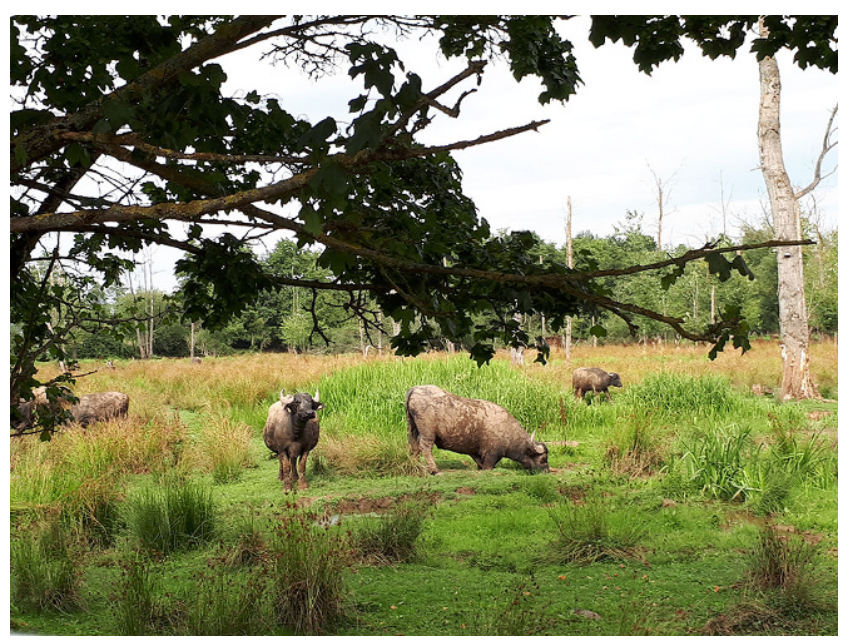

Abb. 3 Wasserbüffel bei Erlensee (ㄷ Christl Wittmann)

Der MKK soll als Wirtschafts-, Arbeits- und Lebensstandort vermarktet und Fachkräfte sollen angesprochen werden. Beim „Marketing für die Region“ (STM 2021, S. 19) geht es in erster Linie um den Aufbau eines Netzwerks mit regionalen Unternehmen zu Kooperationszwecken und um die Vermarktung des MKK im regionalen Standortwettbewerb. Diese Arbeit ist als Ergänzung zur schon vorhandenen Wirtschaftsförderung des Kreises zu sehen, die sich auf ein umfassendes Spektrum von Standortmarketing, wie z.B. Unternehmensansiedlung und Flächenvermarktung, fokussiert. Generell obliegt die Wirtschaftsförderung im MKK aktuell nicht einem einzelnen Akteur: Einzelne Kommunen haben Verantwortliche für dieses Thema (oder Teilbereiche davon). Auch hier sind die Themen Tourismus und Wirtschaftsförderung eng verbunden und teilweise gemeinsam angesiedelt. Die STM strebt mit dem „Marketing für die Region" an, gewisse inspirative Aspekte möglichst MKKweit zu bearbeiten.

\section{Marketing für die Region}

Um den MKK als Wirtschafts-, Arbeits- und Lebensstandort zur vermarkten und zu etablieren, ist die Sicherung von Fachkräften ein wichtiger Aspekt. Hier sind zunehmend weiche Standortfaktoren, wie z. B. Freizeit- oder Wohnwert, von zentraler Bedeutung. Um Bewohner*innen und Fachkräfte für die Region zu gewinnen, muss sich der MKK als eine mögliche Option im umkämpften Standortwettbewerb positionieren und auch als solche wahrgenommen werden. Die DMO soll den MKK nicht mehr nur als Tourismusdestination vermarkten, sondern auch als lebenswerten Landkreis mit guten Möglichkeiten, Freizeit, Familie und Arbeit zu vereinen. Das Zusammenspiel der verschiedenen Aspekte zeigt sich auch am Aufbau der Standortmarketingwebsite www.main-kinzig.com (siehe Abb. 4).

\section{Umsetzung durch die DMO}

Für die Planung und Umsetzung wurde die STM ausgewählt, da die DMO bereits an einem einheitlichen Profil arbeitet. In Anbetracht dieser Tatsache sieht die Standortmarketingstrategie vor, die entwickelte Marketingerfahrung und die inzwischen etablierte Marke „Spessart“ zu nutzen, um eine gemeinsame, konsequente Strategie für ein wettbewerbsfähiges Standortmarketing des MKK zu entwickeln und umzusetzen.

Die Ansiedlung des Standortmarketings unter dem Dach der DMO hat zudem das Ziel, wirtschaftliche Synergien und Mehrwerte im Marketing zu schaffen und somit die Maßnahmen effizient zu gestalten.

Ein dritter Grund für die Ansiedlung der Aufgabe bei der DMO ist die Wechselwirkung mit der Tourismusstrategie. Dort wird als Vision für das Tourismusmarketing u. a. festgehalten: „Der Tourismus trägt ... spürbar zur Standort- und Lebensqualität bei“ (STM 2021, S. 8). Diese Standort- und Lebensqualität soll durch das Standortmarketing hervorgehoben werden, um Fachkräfte für das Wohnen, Leben und Arbeiten im MKK zu gewinnen. Das Standortmarketing baut hierbei auf dem Tourismusmarketing auf: Weiterhin gilt die Tourimusmarketingannahme, dass, was für Gäste von Interesse ist, auch für Bewohner*innen von Interesse ist (z.B. Freizeitmöglichkeiten, Infrastruktur, Ästhetik; Ausnahme: Hotellerie). Durch das STM-Standortmarketing werden allerdings noch zusätzliche Aspekte, wie z.B. Wohnen und Arbeiten im MKK, angesprochen.

Zwar sind die Themen und Strategien für Tourismus und Standortmarketing getrennt angesiedelt, aber durch die Verankerung beider Themen im Verantwortungsbereich der STM werden Grundlagen für Verknüpfungen geschaffen. Maßnahmen können untereinander koordiniert und ggf. abgestimmt werden. Dieses aufeinander abgestimmte Marketing hilft, den MKK nach außen als eine Einheit zu prä- 
LEBEN, WOHNEN UND ARBEITEN IM MAIN-KINZIG-KREIS

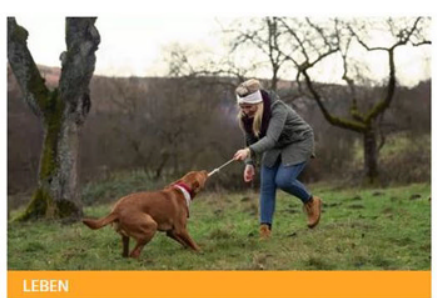

LEBEN IM MAIN-KINZIG-KREIS

Was macht den Main-Kinzig-Kreis so lebenswert? Welche Möglichkeiten gibt es, Deine Freizeit zu gestalten? Wir stellen Dir hier Dein Facettenreich Main-Kinzig-Kreis vor.

(3) Freizeitaktivitäter

(3) Veranstaltungen

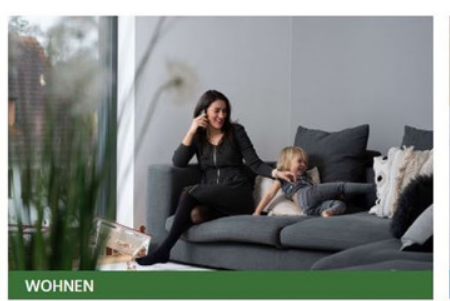

WOHNEN IM MAIN-KINZIG-KREIS

Der Immobilien- und Wohnungsmarkt im Main-KinzigKreis wird aufgrund der Verbindung von Großstadt und Natur immer beliebter. Kann eine der 29 Städte und Gemeinden auch Dein Herz gewinnen?

(1) Gemeinden kennenlernen

(2) Immobilienmarkt

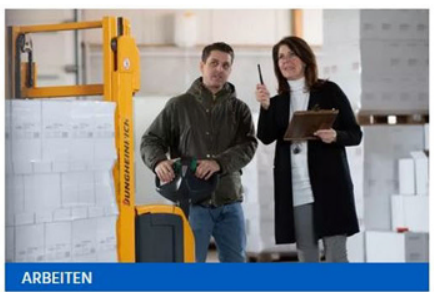

ARBEITEN IM MAIN-KINZIG-KREIS

Karriere machen außerhalb der Metropolen? Ja, das ist möglich. Die Unternehmen im Main-Kinzig-Kreis sind vielseitig und bieten Berufe in den verschiedensten Sektoren an. Auch für Dich wird etwas dabei sein.

(3) Karriere im MKK

Abb. 4 Gliederung der Standortmarketingwebsite. (Quelle: www.main-kinzig.com)

sentieren und nicht als unterschiedliche Welten für Bewohner*innen, Besucher*innen, Arbeitnehmer*innen und Unternehmen. Der ursprüngliche Blick der DMO wird um den Blickwinkel der Neubewohner*innen und Unternehmen erweitert.

\section{Vorteile für den MKK}

Durch die Ansiedlung beider Themen unter dem Dach der STM können gewissen Vorteile entstehen:

- Marketingkompetenz bereits vorhanden (Tourismusmarketing)

- STM bereits mit dem MKK-Gebiet vertraut

- Weiche Standortfaktoren (z.B. Freizeitgestaltung, Lebensqualität)

- Wichtig für Standort- und Tourismusmarketing und STM bekannt

- STM als DMO: Zeigt attraktive Seiten der Region für Besucher*innen und Bewohner*innen auf - Erweiterung auf potenzielle Neu-Bewohner*innen

- Leichtere Abstimmung und Koordination untereinander

- Marke „Spessart“ bereits etabliert

- STM in der Region bekannt - kein neues Unternehmen bzw. neue Kontakte für Standortmarketing-Stakeholder und Politik
Der MKK hat erkannt, dass Tourismusmarketing und weiche Standortfaktoren zur Fachkräfteansprache Hand in Hand gehen und sich durch eine koordinierte, abgestimmte Planung der Maßnahmen Vorteile für die Region ergeben. Synergien können genutzt und Lebensqualität kann gesteigert werden, die Bewohner*innen profitieren zusätzlich von Standortmarketingmaßnahmen und die steigende Lebensqualität und Attraktivität der Region zieht weitere Fachkräfte, Unternehmen und Gäste an. Die Bemühungen im MKK sind noch am Anfang, aber langfristig und mit gesammelten Erfahrungen wird auf Lerneffekte für die $\mathrm{Zu}$ kunft gehofft.

\section{Fazit}

Das übergeordnete Ziel des Lebensraummanagements ist es, die Attraktivität der eigenen Region als Lebens-, Arbeits- aber auch Urlaubsraum zu steigern. Wie aufgezeigt, wird unter diesem Begriff ein breiter Themenkomplex subsumiert, der auch viele tourismusfremde Aufgaben beinhaltet. Wichtig für die DMO ist daher, dass die Umsetzung dieser Themen nicht allein in ihre Verantwortung fällt, sondern ihr die Aufgabe des Netzwerkers und Koordinators zukommt. Die relevanten Aufgaben sind dabei in jeder Destination individuell zu bestimmen und sollten schrittweise in enger Abstimmung mit Bürger*innen und Akteur*innen der Region umgesetzt werden. Entsprechend ist auch eine 
Evaluierung fallspezifisch zu erarbeiten. Diese Individualität erschwert gleichzeitig die Übertragung von Konzepten auf andere Regionen.

Die STM zeigt, wie das in der Praxis funktionieren kann: Dort wurde die Relevanz des Themas erkannt und schrittweise in die Arbeit der DMO integriert, wobei der erste Fokus auf der Gewinnung von Fachkräften und neuen Bewohner*innen liegt. Mit der Ansiedelung bei der DMO sollen Synergien genutzt und die weichen Standortfaktoren der Region hervorgehoben werden.

Aktuelle Diskussionen legen nahe, dass das Lebensraumoder Regionalmanagement die DMO in naher Zukunft verstärkt beschäftigen wird, weshalb eine Auseinandersetzung mit diesem Thema wichtig ist. Insgesamt lässt sich festhalten, dass die Übernahme solcher Aufgaben ein großer Kraftakt für DMO ist, jedoch die Basis für einen nicht nur ökologisch, sondern vor allem auch ökonomisch und sozial nachhaltigen und in der Bevölkerung akzeptierten Tourismus darstellt.

Funding Open Access funding enabled and organized by Projekt DEAL.

Interessenkonflikt C. Wittmann und L. Helleisz geben an, dass kein Interessenkonflikt besteht.

Open Access Dieser Artikel wird unter der Creative Commons Namensnennung 4.0 International Lizenz veröffentlicht, welche die Nutzung, Vervielfältigung, Bearbeitung, Verbreitung und Wiedergabe in jeglichem Medium und Format erlaubt, sofern Sie den/die ursprünglichen Autor(en) und die Quelle ordnungsgemäß nennen, einen Link zur Creative Commons Lizenz beifügen und angeben, ob Änderungen vorgenommen wurden.

Die in diesem Artikel enthaltenen Bilder und sonstiges Drittmaterial unterliegen ebenfalls der genannten Creative Commons Lizenz, sofern sich aus der Abbildungslegende nichts anderes ergibt. Sofern das betreffende Material nicht unter der genannten Creative Commons Lizenz steht und die betreffende Handlung nicht nach gesetzlichen Vorschriften erlaubt ist, ist für die oben aufgeführten Weiterverwendungen des Materials die Einwilligung des jeweiligen Rechteinhabers einzuholen.

Weitere Details zur Lizenz entnehmen Sie bitte der Lizenzinformation auf http://creativecommons.org/licenses/by/4.0/deed.de.

\section{Literatur}

Duden (2021) Lebensraum. https://www.duden.de/rechtschreibung/ Lebensraum. Zugegriffen: 18. Sept. 2021

Fürst D (2013) Destination Governance - ein neues Paradigma in der Steuerung von Destinationen? In: Saretzki A, Wöhler K (Hrsg) Governance von Destinationen. Neue Ansätze für die erfolgreiche Steuerung touristischer Zielgebiete. Erich Schmidt, Berlin, S 19-34
Hölzl B (2019) Destination im Wandel - am Beispiel Meran. In: Pechlaner H (Hrsg) Destination und Lebensraum. Perspektiven touristischer Entwicklung. Springer, Wiesbaden, S 245-255

Kohl \& Partner (2016) Destination 2030; Die Zukunft der touristischen Organisationen. Broschüre

Kohl \& Partner (2020a) Lebensraumkonzept Norderney. https://www. norderney.de/fileadmin/PDF/Lebensraum_Unterlagen/Endbericht_ BER_Lebensraumkonzept_Norderney_200903.pdf. Zugegriffen: 18. Sept. 2021

Kohl \& Partner (2020b) Unser Bad Hindelang 2030; Lebensraumkonzept mit integrierter Tourismusstrategie. https://marktgemeinde. badhindelang.de/fileadmin/Mediendatenbank/PDFs_Gemeinde/ Aktuelles/Lebensraumkonzept_2030/Konzept_Lebensraum_ Hindelang_web.pdf. Zugegriffen: 18. Sept. 2021

Kompetenzzentrum Tourismus des Bundes (2019) Tourismus 2030 - Bausteine der Zukunft. Fakten-Thesen-Perspektiven. https://www.kompetenzzentrum-tourismus.de/images/aktuelles/ Tourismus2030_Web.pdf. Zugegriffen: 16. Dez. 2021

Main-Kinzig-Kreis (2020) Der Main-Kinzig-Kreis in Zahlen. https:// www.mkk.de/landkreis/mkk_in_zahlen_1/mkk_in_zahlen.html. Zugegriffen: 18. Sept. 2021

Milano C, Novelli M, Cheer JM (2019) Overtourism and tourismphobia: a journey through four decades of tourism development, planning and local concerns. Tour Plan Dev 16:353-357

OECD OECD better life index. https://www.oecdbetterlifeindex.org. Zugegriffen: 13. Dez. 2021

Pfeffer-Hoffmann C (2011) Regionale Identität und gesellschaftlicher Zusammenhalt. In: Pfeffer-Hoffmann C, Hendricks W (Hrsg) Generationenübergreifende Entwicklung gesellschaftlicher Perspektiven in der Niederlausitz. Ergebnisse des Projektes Anstoß. Centaurus, Freiburg, S 11-22

Regionalmarke EIFEL Eine Region setzt ein Zeichen. https://www. regionalmarke-eifel.de/regionalmarke/philosophie. Zugegriffen: 19. Dez. 2021

Seeler S, Eisenstein B, Müller S (2021) Tourismusakzeptanz in der Wohnbevölkerung - Vorstellung der Studie, Kernergebnisse auf Bundesebene \& Beteiligungsmöglichkeiten. https://www.ditffhw.de/fileadmin/content/downloads/aktuelle_projekte/DITF DTV_Vorstellung_Tourismusakzeptanz_der_Wohnbevoelkerung. pdf. Zugegriffen: 18. Sept. 2021

Spessart Tourismus und Marketing GmbH (STM) (2021) Fortschreibung des Strategischen Entwicklungsplans - Für den Tourismus in der Destination Spessart. https://www.hessen.tourismusnetzwerk. info/wp-content/uploads/2021/06/20210519_Fortschreibung _ Strategie.pdf. Zugegriffen: 18. Sept. 2021

Spessart Tourismus und Marketing GmbH (STM) (2017) Strategischer Entwicklungsplan - Für den Tourismus in der Destination Spessart. https://www.spessart-tourismus.de/files/spessart-tourismus/ downloads/2017_07_13_Strategischer\%20Entwicklungsplan Spessart_Web.pdf. Zugegriffen: 18. Sept. 2021

Steinecke A, Herntrei M (2017) Destinationsmanagement. UVK/ Lucius, Konstanz, München

Uğur L (2017) Mind the gap: reconceptualising inclusive development in support of integrated Urban planning and tourism development. In: Bellini N, Pasquinelli C (Hrsg) Tourism in the city. Springer, Cham, S 51-66

World Health Organization (1997) WHOQOL: Measuring quality of life. https://apps.who.int/iris/handle/10665/63482. Zugegriffen: 18. Sept. 2021 